\title{
Dynamical characteristics of asphalt binder containing Polyphosphoric acid modifiers
}

\author{
YE Qun Shan ${ }^{1,2, a^{*}}$ \\ ${ }^{1}$ Communication and Transportation Engineering, Changsha University of Science and Technology, \\ Changsha 410004, China \\ ${ }^{2}$ State Key Laboratory of Silicate Materials for Architectures (Wuhan University of Technology), \\ wuhan 430070, China \\ ayeqs@whut.edu.cn
}

Keywords: Polyphosphoric acid; modified asphalt; complex modulus; phase angle; rutting parameter.

Abstract. The rheological properties of Polyphosphoric acid (PPA) modified asphalt binder were investigated by the dynamic shear test, and the composition change of modified asphalt was use to analyze the improvement mechanism of rheological characteristics. Test results indicated that the complex shear modulus of PPA modified asphalt binder was decreased at low temperatures, while the phase angle of asphalt binder was increased. At high temperatures, the complex shear modulus of asphalt binder with PPA modifier was increased while the phase angle was decreased. The high temperature grade of PPA modified asphalt binder could be increased 1 2 levels when the content of PPA was up to $1.0 \sim 1.5 \%$, which implied that the rutting resistance of PPA modified asphalt binder was improved significantly.

\section{Introduction}

The use of modified asphalt binders has grown tremendously in the road construction. This is due to the increased stress on the highways from higher traffic volumes and heavier loads. Many different modifiers have been used to improve the binder properties to better meet specifications. These modifiers include both organic and inorganic material such as styrene butadiene (SB), styrene-butadiene-styrene (SBS), ethylene terpolymer, and Polyphosphoric acid (PPA). Concerns have been raised by some researchers about the actual performance characteristics of the last polymer, PPA. These concerns include reversibility of the stiffening effect of PPA on asphalt binders and interactions with other additives in the mixtures such as hydrated lime[1]. Several grades of phosphoric acid containing different amounts of orthophosphoric acid are commercially available. They contain 50\% ("green acid"), 75\%, 85\% and 100\% orthophosphoric acid. Two other grades superphosphoric and PPA contain 105\% and 115\% orthophosphoric acid and are mixtures of pyrophosphoric acid, triphosphoric, and higher acids.

Baldino employed Dynamic Mechanical Analysis(DMA) to investigate the effects of PPA on bitumen from different sources at low temperatures, test results indicated that the PPA seems to improve low temperature bitumen behavior and an optimal PPA concentration and be obtained[2]. Edwards studied the rheological effects of commercial waxes and PPA in bitumen 160/220 at high and medium temperature, which found that considerable positive effects on the rheological behavior could be achieved when adding polyethylene wax or PPA especially to a non-waxy bitumen[3].

PPA modified asphalt binder and mixture have been successfully applied in construction field [4-6]. However, the effect of PPA additives on the pavement engineering performances is profound, and the mechanism of PPA affect bitumen is complex. The principle objectives of this research are to investigate the rheological characteristics of PPA modified asphalt binders and the effects of such modifier on the complex modulus, phase angle and rutting parameters are analyzed. 


\section{Raw materials and test procedure}

A base bitumen (A-70), provided by Tipco Asphalt Public Co. Ltd was used for PPA modification. A SBS modified asphalt binder provided by the company was used to compare the PPA modified asphalt. The properties of such asphalt binders are presented in Table 1 in conformity with the relevant test methods. PPA modifier was provided by Aladdin Reagent (Shanghai, China).

Table 1 Properties of the base bitumen and SBS modified asphalt

\begin{tabular}{lccc}
\hline \multicolumn{1}{c}{ Items } & Specification & A-70 & SBS modified \\
\hline Penetration $\left(25^{\circ} \mathrm{C} ; 0.1 \mathrm{~mm}\right)$ & ASTM D-5 & 67.5 & 52.5 \\
Softening point $\left({ }^{\circ} \mathrm{C}\right)$ & ASTM D-36 & 50.5 & 78.9 \\
Ductility $\left(25^{\circ} \mathrm{C}\right)$ & ASTM D-113 & $100+$ & 31.5 \\
Flash point $\left({ }^{\circ} \mathrm{C}\right)$ & ASTM D-92 & 300 & 300 \\
Viscosity $\left(135^{\circ} \mathrm{C} ; \mathrm{Pa} \cdot \mathrm{s}\right)$ & ASTM D-4402 & 467 & 2265 \\
\hline
\end{tabular}

The PPA modified asphalt binder was obtained by a constant mixer at $165^{\circ} \mathrm{C}$. The PPA concentrations were $0.5,1.0$ and 1.5 percent by weight of the bitumen. To obtain homogenous bitumen-fiber mastics, the PPA modifier were added slowly into the preheated base bitumen and mixing for two hours.

Complex shear modulus and phase angle of fiber modified asphalt binders were obtained using an Anton Paar Physical MCR 501 Rheometer. Temperature sweep tests were conducted over a range of test temperatures from $-10{ }^{\circ} \mathrm{C}$ to $80{ }^{\circ} \mathrm{C}$ with the frequency of $10 \mathrm{rad} / \mathrm{s}$.

\section{Results and discussion}

(1) Complex shear modulus

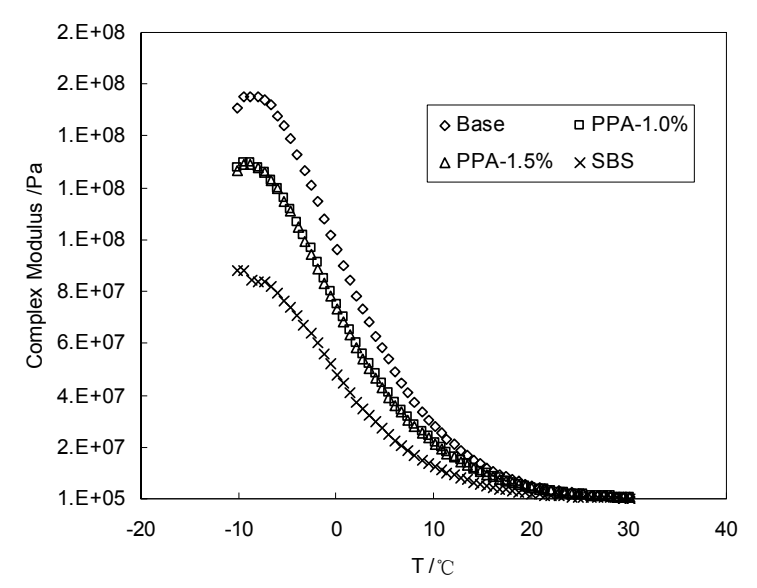

Fig. 1 Effect of PPA on the complex modulus of asphalt binders $\left(-10^{\circ} \mathrm{C} \sim 30^{\circ} \mathrm{C}\right)$



Fig.2 Effect of PPA on the complex modulus of asphalt binders $\left(30^{\circ} \mathrm{C} \sim 80^{\circ} \mathrm{C}\right)$

The complex shear modulus of PPA and PPA/SBS modified asphalt binders with different concentrations are shown in Fig.1 Fig.2, and compared with the base asphalt and SBS modified asphalt at different temperatures. It could be found from Fig. 1 that the complex shear modulus of asphalt modified was decreased violently when the PPA or SBS modifier added, which implied that the toughness of asphalt binder were enhanced, and the resistance to crack of asphalt could be improved at such lower temperatures (from $-10^{\circ} \mathrm{C}$ to $30^{\circ} \mathrm{C}$ ). Furthermore, the improvement of PPA modifier was weaker when compared with SBS modifier. The decrease trend of complex shear modulus was vanished gradually with increasing temperature for modified asphalt binders. The complex shear modulus with the PPA concentration of $1.0 \%$ or $1.5 \%$ were similar with the base asphalt at temperature of $25^{\circ} \mathrm{C}$. 
The complex shear modulus of asphalt binders were shown in Fig.2. It indicates that the complex shear modulus of asphalt binders was increased by the addition of PPA, which resulted in the enhancement of stiffness for asphalt binders. The stiffen effect presented more significant with the increase of PPA concentration and became gently when the PPA concentration was up to $1.0 \%$. It also could be found that the stiffen effect of PPA represented more significantly than the SBS modifier.

(2) Phase angle

Phase angles of asphalt binders modified by the PPA were shown in Fig. 3 and Fig.4. The phase angles of asphalt binders with PPA or SBS modifiers were increased at low temperatures, which could be found in Fig.3. The phase angle of PPA modified asphalt was throughout higher than that of base asphalt when the test temperature was below $8{ }^{\circ} \mathrm{C}$, and for the SBS modified asphalt the temperature was below $20{ }^{\circ} \mathrm{C}$. It implied that the viscous port and elastic port of asphalt binder were increased and decreased respectively when the PPA modifier was used, which was more positive for the resistance to crack at low temperatures for asphalt binder. When the test temperatures was at $30 \sim 80{ }^{\circ} \mathrm{C}$ range, the phase angles of PPA modified asphalt binders were reduced greatly, which indicated that the elastic portion in viscoelastic property of asphalt binders was increased. The reduction of phase angles could be up to $10 \sim 20^{\circ}$ for asphalt binder containing $1.0 \%$ PPA modifiers at different temperatures.

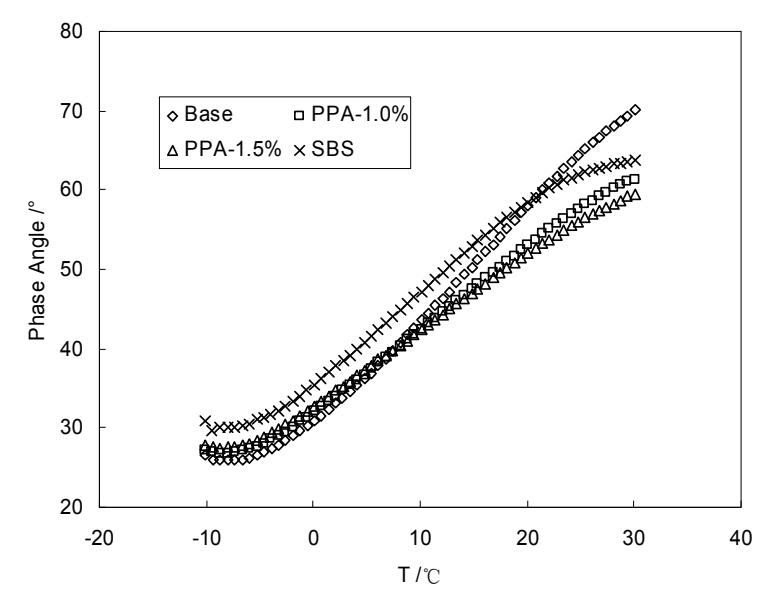

Fig.3 Effect of PPA on the phase angle of asphalt binders $\left(-10 \sim 30^{\circ} \mathrm{C}\right)$

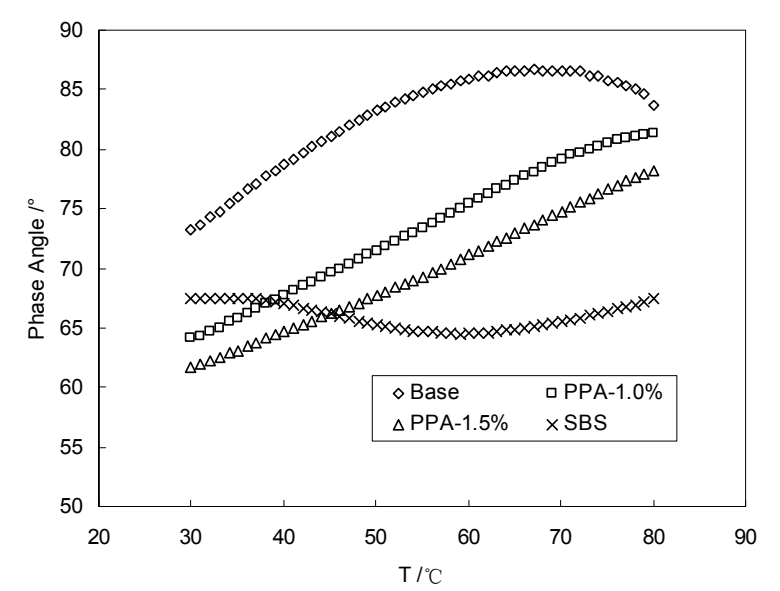

Fig.4 Effect of PPA on the phase angle of asphalt binders $\left(30 \sim 80^{\circ} \mathrm{C}\right)$

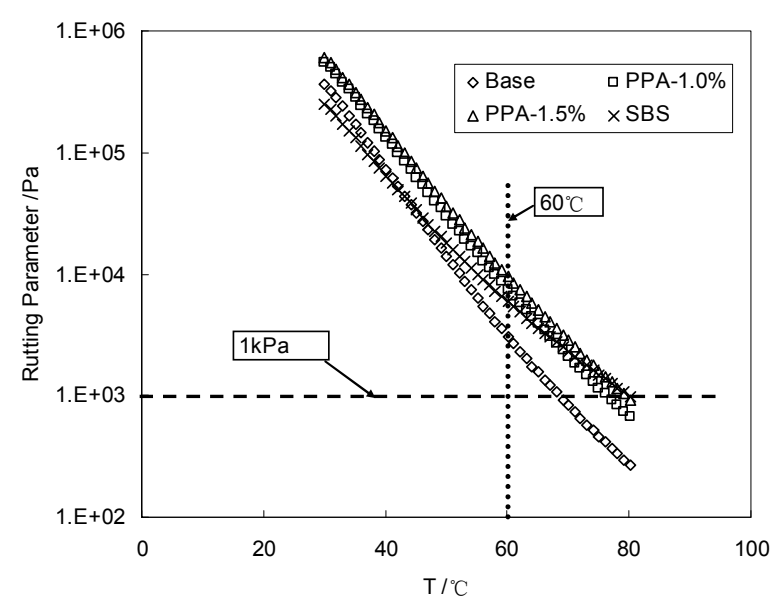

Fig.5 Rutting parameters of asphalt binder with different modifiers

(3) Rutting parameters

Rutting parameter was adopted to evaluate the rutting resistance of PPA modified asphalt binder at high temperatures, and compared with that of SBS modified asphalt binder. It could be found in Fig.5 
that the rutting parameter was increased for PPA modified asphalt binder in the whole temperature range, which indicated that the rutting resistance of asphalt binder was improved. The increment of rutting parameter became more significantly when the concentration of PPA and the test temperature were increased. When compared with the base asphalt at temperature of $60^{\circ} \mathrm{C}$, the increment of rutting parameter was 1.4 fold and 2.1 fold for the modified asphalt with PPA concentration of $1.0 \%$ and $1.5 \%$ respectively, it was even higher than that of SBS modified asphalt binder.

Moreover, the critical temperature $\mathrm{T}(=1.0 \mathrm{kpa})$ was also employed to evaluate the high temperature stability of asphalt binder. It could be found in Fig. 5 that the critical temperatures for base asphalt, asphalt with $1.0 \%$ PPA, asphalt with $1.5 \%$ PPA and SBS modified asphalt were $68.6^{\circ} \mathrm{C}$, $76.4^{\circ} \mathrm{C}, 79.4^{\circ} \mathrm{C}$ and $79.8^{\circ} \mathrm{C}$ respectively. It approved that the high temperature grade of asphalt could be increased 1 2 levels with suitable PPA concentration. The critical temperature for asphalt with $1.5 \%$ PPA was correspondent to that of SBS modified asphalt binder.

\section{Conclusion}

Based on the results presented in this paper, the following conclusions can be drawn.

(1) The complex shear modulus of PPA modified asphalt binder was decreased at low temperatures, while the phase angle of asphalt binder was increased. It implied that the toughness and resistance to cracking for PPA modified asphalt were improved.

(2) At high temperatures, the complex shear modulus of asphalt binder with PPA modifier was increased while the phase angle was decreased. It approved that the stiffness and the elastic properties of asphalt binder could be enhanced by the addition of PPA modifier, which indicated that the resistance to deformation was improved for asphalt binder.

(3) Rutting parameter of asphalt binder could be increased significantly by the use of PPA modifier. The critical temperature of modified asphalt binder was increased 1 2 levels when the concentration of PPA modifier at the range of $1.0 \sim 1.5 \%$.

\section{Acknowledgements}

This work was supported by the National Key Scientific Apparatus Development Program from the Ministry of Science and Technology of China (No. 2013YQ160501) and State Key Laboratory of Silicate Materials for Architectures (Wuhan University of Technology)(Project No. SYSJJ2013-02).

\section{References}

[1] Kodrat I, Sohn D, Hesp S A M. Comparison of Polyphosphoric acid-modified asphalt binders with straight and polymer-modified materials [J]. Transportation Research Record, 1998(2007): 47-55.

[2] Baldino N, Gabriele D, Oliviero Rossi C, et al,. Low temperature rheology of Polyphosphoric acid (PPA) added bitumen. Construction and Building Materials, 36(2012): 592-596.

[3] Edwards Y, Tasdemir Y, Isacsson U. Rheological effects of commercial waxes and Polyphosphoric acid in bitumen 160/220-high and medium temperature performance [J]. Construction and Building Materials, 21(2007): 1899-1908.

[4] Masson J-F, Gagne M. Polyphosphoric acid (PPA)-modified bitumen: disruption of the asphaltenes network based on the reaction of non-basic nitrogen with PPA. Energy Fuels, 22(2008): 3402-3406.

[5] Masson J-F, Collins P. FTIR study of the reaction of Polyphosphoric acid and model bitumen sulfur compounds. Energy Fuels, 23(2009): 440-442.

[6] Baldino N, Gabriele D, Oliviero Rossi C, et al,. Low temperature rheology of Polyphosphoric acid (PPA) added bitumen. Construction and Building Materials, 36(2012): 592-596. 